\title{
Existence results for fractional functional integrodifferential equations with nonlocal conditions in Banach spaces
}

\author{
by ZuOMAO YAN (Zhangye)
}

\begin{abstract}
The paper establishes a sufficient condition for the existence of mild solutions of fractional functional integrodifferential equations with nonlocal conditions in Banach spaces. Our approach is based on Schaefer's fixed point theorem combined with the use of strongly continuous operator semigroups. As an application, we also consider a fractional partial functional integrodifferential equation.
\end{abstract}

1. Introduction. In this paper, we consider the existence of mild solutions for the fractional functional integrodifferential equation with nonlocal condition of the form

$$
\begin{aligned}
& D^{\beta} x(t)=A x(t) \\
& +F\left(t, x\left(\sigma_{1}(t)\right), \ldots, x\left(\sigma_{n}(t)\right), \int_{0}^{t} h\left(t, s, x\left(\sigma_{n+1}(s)\right)\right) d s\right), \quad t \in J, \\
& x(0)+g(x)=x_{0},
\end{aligned}
$$

where $J=[0, b], 0<\beta<1, D^{\beta}$ is the standard Riemann-Liouville fractional derivative, the state $x(\cdot)$ takes values in a Banach space $X$ with the norm $\|\cdot\|$ and $A$ generates a strongly continuous semigroup $T(t)$ in $X$. The nonlinear operators $F: J \times X^{n+1} \rightarrow X, h: J \times J \times X \rightarrow X, g: C(J, X) \rightarrow X$, $\sigma_{i}: J \rightarrow J, i=1, \ldots, n+1$, are given functions.

Differential equations of fractional order play an important role in describing some real world problems. This is caused both by the intensive development of the theory of fractional calculus itself and by applications of such constructions in various domains of science, such as physics, mechanics, chemistry, engineering, etc. For details, see [DF], [ES], [H], [M], [MR], [P],

2010 Mathematics Subject Classification: Primary 34K37, 34K30; Secondary 34A08, $34 \mathrm{G} 20$.

Key words and phrases: fractional functional integrodifferential equation, fractional derivative, fixed point, nonlocal condition. 
[SK] and references therein. Recently, much attention has been paid to existence results for differential equations with fractional order, for example, El-Sayed and Ibrahim [EI], Benchohra et al. [BH], Lakshmikantham and Vastala [L], [LV], and Yu and Gao [YG]. Some classes of evolution equations have been considered by El-Borai [E] and Muslim [M]. In [JA], the authors have studied the existence and uniqueness of mild solutions for the initial value problem for a semilinear integrodifferential equation involving Caputo's fractional derivative.

On the other hand, the first results concerning the existence and uniqueness of mild solutions to abstract Cauchy problems with nonlocal initial conditions were formulated and proved by Byszewski; see [B], BL]. Subsequently, many authors studied nonlocal Cauchy problems because they turned out to be better suited to some applications than the classical Cauchy problems. For example, see Deng [D], Byszewski and Akca [BA], Ntouyas and Tsamatos [NT], Lin and Liu [LL], Liang et al. [LX], Aizicovici and McKibben [AM], G. M. N'Guérékata [N], and Mophou and N'Guérékata $[\mathrm{MN}]$, MG]. Very recently, Balachandran and Park [BP] have proved the existence and uniqueness of mild solutions to the abstract nonlocal Cauchy problem for fractional differential equations in Banach space. The results are obtained by using the contraction mapping principle and the Krasnosel'skil fixed point theorem. In this paper, we shall investigate the existence of mild solutions of the nonlocal Cauchy problem (1.1)-(1.2) in Banach spaces by a different method, using the semigroup theory, the Banach contraction principle and Schaefer's fixed point theorem.

This paper is organized as follows. In Section 2, we briefly recall some basic definitions and preliminary facts. Section 3 is devoted to the existence of mild solutions of problem (1.1)-(1.2). Finally, a concrete example is presented in Section 4 to show the application of our main results.

2. Preliminaries. In this section, we introduce some basic definitions and lemmas which are used throughout this paper.

Let $C(J, X)$ denote the Banach space of continuous functions from $J$ into $X$ with the norm

$$
\|x\|_{\infty}=\sup \{\|x(t)\|: t \in J\}
$$

and let $L(X)$ denote the Banach space of bounded linear operators from $X$ to $X$.

A measurable function $x: J \rightarrow X$ is Bochner integrable if and only if $\|x\|$ is Lebesgue integrable (for properties of the Bochner integral see Yosida [Y]). $L^{1}(J, X)$ denotes the Banach space of measurable functions $x: J \rightarrow X$ which 
are Bochner integrable normed by

$$
\|x\|_{L^{1}}=\int_{0}^{b}\|x(t)\| d t \quad \text { for all } x \in L^{1}(J, X) .
$$

Now, we recall some definitions and facts about fractional derivatives and fractional integrals of arbitrary orders (see $[\mathrm{H}],[\mathrm{MR},[\mathrm{P},[\mathrm{SK}]$ ).

Definition 2.1. The fractional primitive of order $\beta>0$ of a function $h:(0, b] \rightarrow X$ is defined by

$$
I_{0}^{\beta} h(t)=\int_{0}^{t} \frac{(t-s)^{\beta-1}}{\Gamma(\beta)} h(s) d s,
$$

provided the right hand side exists pointwise on $(0, b]$, where $\Gamma$ is the gamma function.

For instance, $I^{\beta} h$ exists for all $\beta>0$ when $h \in C((0, b], X) \cap L^{1}((0, b], X)$; note also that if $h \in C([0, b], X)$ then $I^{\beta} h \in C([0, b], X)$, and moreover $I^{\beta} h(0)=0$.

Definition 2.2. The fractional derivative of order $\beta>0$ of a continuous function $h:(0, b] \rightarrow X$ is given by

$$
\frac{d^{\beta} h(t)}{d t^{\beta}}=\frac{1}{\Gamma(1-\beta)} \frac{d}{d t} \int_{0}^{t}(t-s)^{\beta-1} h(s) d s=\frac{d}{d t} I_{0}^{\beta} h(t) .
$$

Definition 2.3. A continuous function $x(\cdot): J \rightarrow X$ is called a mild solution of the problem (1.1)-(1.2) if for all $x_{0} \in X$, it satisfies the following integral equation:

$$
\begin{aligned}
& x(t)=T(t)\left[x_{0}-g(x)\right]+\frac{1}{\Gamma(\beta)} \int_{0}^{t}(t-s)^{\beta-1} \\
& \quad \times T(t-s) F\left(s, x\left(\sigma_{1}(s)\right), \ldots, x\left(\sigma_{n}(s)\right), \int_{0}^{s} h\left(s, \tau, x\left(\sigma_{n+1}(\tau)\right)\right) d \tau\right) d s .
\end{aligned}
$$

LEMMA 2.4 (Schaefer's fixed point theorem $[\overline{D G}]$ ). Let $E$ be a normed linear space. Let $Q: E \rightarrow E$ be a completely continuous operator, that is, it is continuous and the image of any bounded set is contained in a compact set and let

$$
\zeta(Q)=\{x \in E: x=\lambda Q x \text { for some } 0<\lambda<1\} .
$$

Then either $\zeta(Q)$ is unbounded or $Q$ has a fixed point.

Further we assume the following hypotheses: 
(H1) $T(t), t>0$, is a compact semigroup and there exists a constant $M \geq 1$ such that

$$
\|T(t)\| \leq M
$$

(H2) The function $F: J \times X^{n+1} \rightarrow X$ is continuous and there exist constants $L, L_{1}>0$ such that for all $x_{i}, y_{i} \in X, i=1, \ldots, n+1$,

$$
\left\|F\left(t, x_{1}, \ldots, x_{n+1}\right)-F\left(t, y_{1}, \ldots, y_{n+1}\right)\right\| \leq L\left[\sum_{i=1}^{n+1}\left\|x_{i}-y_{i}\right\|\right]
$$

and

$$
L_{1}=\max _{t \in J}\|F(t, 0, \ldots, 0)\| .
$$

(H3) The function $h: J \times J \times X \rightarrow X$ is continuous and there exist constants $N, N_{1}>0$ such that for all $x, y \in X$,

$$
\|h(t, s, x)-h(t, s, y)\| \leq N\|x-y\|
$$

and

$$
N_{1}=\max _{0 \leq s \leq t \leq b}\|h(t, s, 0)\| .
$$

(H4) $\sigma_{i}: J \rightarrow J, i=1, \ldots, n+1$, are continuous functions such that $\sigma_{i}(t) \leq t, i=1, \ldots, n+1$.

(H5) The function $g(\cdot): C(J, X) \rightarrow X$ is continuous and there exists a $\delta \in(0, b)$ such that $g(\phi)=g(\psi)$ for any $\phi, \psi \in C:=C(J, X)$ with $\phi=\psi$ on $[\delta, b]$.

(H6) There is a constant $c>0$ such that

$$
0 \leq \limsup _{\|\phi\| \rightarrow \infty} \frac{\|g(\phi)\|}{\|\phi\|} \leq c, \quad \phi \in C,
$$

and

$$
M c e^{M L(n+N b) b^{\beta} / \Gamma(\beta+1)}<1 .
$$

\section{Main results}

THEOREM 3.1. If the assumptions $(\mathrm{H} 1)-(\mathrm{H} 6)$ are satisfied, then the nonlocal Cauchy problem (1.1)-(1.2) has at least one mild solution on J.

Proof. Let $L_{0}>0$ be a constant such that

$$
q:=\sup _{t \in J} \frac{L M(n+N b)}{\Gamma(\beta)} \int_{0}^{t} e^{-L_{0}(t-s)}(t-s)^{\beta-1} d s<1,
$$

and we introduce in the space $C(J, X)$ an equivalent norm defined as

$$
\|\phi\|_{V}:=\sup _{t \in J} e^{-L_{0} t}\|\phi(t)\| .
$$


Then it is easy to see that $V:=\left(C(J, X),\|\cdot\|_{V}\right)$ is a Banach space. Fix $v \in C(J, X)$ and for $t \in J$ and $\phi \in V$, define

$$
\begin{aligned}
& \left(Q_{v} \phi\right)(t) \\
& =T(t)\left[x_{0}-g(v)\right]+\frac{1}{\Gamma(\beta)} \int_{0}^{t}(t-s)^{\beta-1} \\
& \quad \times T(t-s) F\left(s, \phi\left(\sigma_{1}(s)\right), \ldots, \phi\left(\sigma_{n}(s)\right), \int_{0}^{s} h\left(s, \tau, \phi\left(\sigma_{n+1}(\tau)\right)\right) d \tau\right) d s .
\end{aligned}
$$

Since $T(\cdot)\left(x_{0}-g(v)\right) \in C(J, X)$, it follows from (H1)-(H4) that $\left(Q_{v} \phi\right)(t)$ $\in V$ for all $\phi \in V$. Let $\phi, \psi \in V$. We have

$$
\begin{aligned}
e^{-L_{0} t} & \left\|\left(Q_{v} \phi\right)(t)-\left(Q_{v} \psi\right)(t)\right\| \\
\leq & \frac{e^{-L_{0} t}}{\Gamma(\beta)} \int_{0}^{t}(t-s)^{\beta-1} \\
& \times \| T(t-s)\left[F\left(s, \phi\left(\sigma_{1}(s)\right), \ldots, \phi\left(\sigma_{n}(s)\right), \int_{0}^{s} h\left(s, \tau, \phi\left(\sigma_{n+1}(\tau)\right)\right) d \tau\right)\right. \\
& \left.-F\left(s, \psi\left(\sigma_{1}(s)\right), \ldots, \psi\left(\sigma_{n}(s)\right), \int_{0}^{s} h\left(s, \tau, \psi\left(\sigma_{n+1}(\tau)\right)\right) d \tau\right)\right] \| d s \\
\leq & \frac{L M}{\Gamma(\beta)} \int_{0}^{t} e^{-L_{0} t}(t-s)^{\beta-1}\left[\left\|\phi\left(\sigma_{1}(s)\right)-\psi\left(\sigma_{1}(s)\right)\right\|\right. \\
& +\cdots+\left\|\phi\left(\sigma_{n}(s)\right)-\psi\left(\sigma_{n}(s)\right)\right\| \\
& \left.+\left\|\int_{0}^{s} h\left(s, \tau, \phi\left(\sigma_{n+1}(\tau)\right)\right) d \tau-\int_{0}^{s} h\left(s, \tau, \psi\left(\sigma_{n+1}(\tau)\right)\right) d \tau\right\|\right] d s \\
\leq & \frac{L M}{\Gamma(\beta)} \int_{0}^{t} e^{-L_{0} t}(t-s)^{\beta-1}[\|\phi(s)-\psi(s)\|+\cdots+\|\phi(s)-\psi(s)\| \\
& \left.+N \int_{0}^{s}\left\|\phi\left(\sigma_{n+1}(\tau)\right)-\psi\left(\sigma_{n+1}(\tau)\right)\right\| d \tau\right] d s \\
\leq & \frac{L M}{\Gamma(\beta)} \int_{0}^{t} e^{-L_{0} t}(t-s)^{\beta-1}[n\|\phi(s)-\psi(s)\|+N b\|\phi(s)-\psi(s)\|] d s \\
\leq & \frac{L M}{\Gamma(\beta)} \int_{0}^{t} e^{-L_{0}(t-s)}(t-s)^{\beta-1}\left[n e^{-L_{0} s}\|\phi(s)-\psi(s)\|\right. \\
& \left.+N b \sup _{s \in J} e^{-L_{0} s}\|\phi(s)-\psi(s)\|\right] d s \\
\leq & \frac{L M(n+N b)}{\Gamma(\beta)} \int_{0}^{t} e^{-L_{0}(t-s)}(t-s)^{\beta-1} d s\|\phi-\psi\|_{V} \leq q\|\phi-\psi\|_{V}, \quad t \in J .
\end{aligned}
$$


Thus

$$
\left\|Q_{v} \phi-Q_{v} \psi\right\|_{V} \leq q\|\phi-\psi\|_{V}, \quad \phi, \psi \in V .
$$

Therefore, $Q_{v}$ is a strict contraction. By the Banach contraction principle, $Q_{v}$ has a unique fixed point $\phi_{v} \in V$ and equation (3.1) has a unique mild solution on $[0, b]$. Set

$$
\widetilde{v}(t):= \begin{cases}v(t) & \text { if } t \in(\delta, b], \\ v(\delta) & \text { if } t \in[0, \delta]\end{cases}
$$

From (3.1), we have

$$
\begin{aligned}
& \phi_{\widetilde{v}}(t)=T(t)\left[x_{0}-g(\widetilde{v})\right]+\frac{1}{\Gamma(\beta)} \int_{0}^{t}(t-s)^{\beta-1} \\
& \times T(t-s) F\left(s, \phi_{\widetilde{v}}\left(\sigma_{1}(s)\right), \ldots, \phi_{\widetilde{v}}\left(\sigma_{n}(s)\right), \int_{0}^{s} h\left(s, \tau, \phi_{\widetilde{v}}\left(\sigma_{n+1}(\tau)\right)\right) d \tau\right) d s .
\end{aligned}
$$

Consider the map $P: C_{\delta}=C([\delta, b], X) \rightarrow C_{\delta}$ defined by

$$
(P v)(t)=\phi_{\widetilde{v}}(t), \quad t \in[\delta, b] .
$$

We shall show that $P$ satisfes all conditions of Lemma 2.4. The proof will be given in several steps.

STEP 1. The set $\Omega=\left\{v \in C_{\delta}: v=\lambda P(v)\right.$ for some $\left.\lambda \in(0,1)\right\}$ is bounded.

Indeed, let $v \in C_{\delta}$ be a possible solution of $v=\lambda P(v)$ for some $0<\lambda<1$. This implies, by (3.2) and (3.3), that for each $t \in(0, b]$,

$$
\begin{aligned}
& v(t)=\lambda \phi_{\widetilde{v}}(t)=\lambda T(t)\left[x_{0}-g(\widetilde{v})\right]+\lambda \frac{1}{\Gamma(\beta)} \int_{0}^{t}(t-s)^{\beta-1} \\
& \times T(t-s) F\left(s, \phi_{\widetilde{v}}\left(\sigma_{1}(s)\right), \ldots, \phi_{\widetilde{v}}\left(\sigma_{n}(s)\right), \int_{0}^{s} h\left(s, \tau, \phi_{\widetilde{v}}\left(\sigma_{n+1}(\tau)\right)\right) d \tau\right) d s .
\end{aligned}
$$

From (H6), there exist positive constants $\epsilon$ and $\gamma$ such that, for all $\|\phi\|>\gamma$,

$$
\|g(\phi)\| \leq(c+\epsilon)\|\phi\|, \quad M(c+\epsilon) e^{M L(n+N b) b^{\beta} / \Gamma(\beta+1)}<1 .
$$

Let

$$
\begin{aligned}
& E_{1}=\{\phi:\|\phi\| \leq \gamma\}, \quad E_{2}=\{\phi:\|\phi\|>\gamma\} \\
& C_{1}=\max \left\{\|g(\phi)\|: \phi \in E_{1}\right\}
\end{aligned}
$$

Thus,

$$
\|g(\phi)\| \leq C_{1}+(c+\epsilon)\|\phi\|
$$


By (H1)-(H4) and (3.6), from (3.4) we have for each $t \in(0, b],\|v(t)\| \leq$ $\left\|\phi_{\widetilde{v}}(t)\right\|$ and

$$
\begin{aligned}
& \left\|\phi_{\widetilde{v}}(t)\right\| \leq\left\|T(t)\left[x_{0}-g(\widetilde{v})\right]\right\|+\frac{1}{\Gamma(\beta)} \int_{0}^{t}(t-s)^{\beta-1} \\
& \times\left\|T(t-s) F\left(s, \phi_{\widetilde{v}}\left(\sigma_{1}(s)\right), \ldots, \phi_{\widetilde{v}}\left(\sigma_{n}(s)\right), \int_{0}^{s} h\left(s, \tau, \phi_{\widetilde{v}}\left(\sigma_{n+1}(\tau)\right)\right) d \tau\right)\right\| d s \\
& \leq M\left[\left\|x_{0}+g(\widetilde{v})\right\|\right]+\frac{M}{\Gamma(\beta)} \int_{0}^{t}(t-s)^{\beta-1} \\
& \times\left\|F\left(s, \phi_{\widetilde{v}}\left(\sigma_{1}(s)\right), \ldots, \phi_{\widetilde{v}}\left(\sigma_{n}(s)\right), \int_{0}^{s} h\left(s, \tau, \phi_{\widetilde{v}}\left(\sigma_{n+1}(\tau)\right)\right) d \tau\right)\right\| d s \\
& \leq M\left[\left\|x_{0}+g(\widetilde{v})\right\|\right]+\frac{M}{\Gamma(\beta)} \int_{0}^{t}(t-s)^{\beta-1} \\
& \times\left[\| F\left(s, \phi_{\widetilde{v}}\left(\sigma_{1}(s)\right), \ldots, \phi_{\widetilde{v}}\left(\sigma_{n}(s)\right), \int_{0}^{s} h\left(s, \tau, \phi_{\widetilde{v}}\left(\sigma_{n+1}(\tau)\right)\right) d \tau\right)\right. \\
& -F(s, 0, \ldots, 0)\|+\| F(s, 0, \ldots, 0) \|] d s \\
& \leq M\left[\left\|x_{0}\right\|+\|g(\widetilde{v})\|\right]+\frac{M}{\Gamma(\beta)} \int_{0}^{t}(t-s)^{\beta-1} \\
& \times\left\{L \left[\left\|\phi_{\widetilde{v}}(s)\right\|+\cdots+\left\|\phi_{\widetilde{v}}(s)\right\|+\int_{0}^{s}\left[\| h\left(s, \tau, \phi_{\widetilde{v}}\left(\sigma_{n+1}(\tau)\right)\right)\right.\right.\right. \\
& \left.-h(s, \tau, 0)\|+\| h(s, \tau, 0) \|] d \tau]+L_{1}\right\} d s \\
& \leq M\left[\left\|x_{0}\right\|+C_{1}+(c+\epsilon)\|\widetilde{v}\|\right]+\frac{M}{\Gamma(\beta)} \int_{0}^{t}(t-s)^{\beta-1} \\
& \times\left\{L\left[n\left\|\phi_{\widetilde{v}}(s)\right\|+b\left(N\left\|\phi_{\widetilde{v}}(s)\right\|+N_{1}\right)\right]+L_{1}\right\} d s \\
& \leq M^{*}+M(c+\epsilon)\|\widetilde{v}\|+\frac{M L(n+N b)}{\Gamma(\beta)} \int_{0}^{t}(t-s)^{\beta-1}\left\|\phi_{\widetilde{v}}(s)\right\| d s,
\end{aligned}
$$

where $M^{*}=M\left[\left\|x_{0}\right\|+C_{1}\right]+M b^{\beta}\left(b L N_{1}+L_{1}\right) / \Gamma(\beta+1)$. Using the Gronwall inequality, we get

$$
\left\|\phi_{\widetilde{v}}(t)\right\| \leq\left[M^{*}+M(c+\epsilon)\|\widetilde{v}\|\right] e^{M L(n+N b) b^{\beta} / \Gamma(\beta+1)} .
$$

Consequently,

$$
\|v\| \leq\left[M^{*}+M(c+\epsilon)\|\widetilde{v}\|\right] e^{M L(n+N b) b^{\beta} / \Gamma(\beta+1)},
$$


and therefore

$$
\|v\| \leq \frac{M^{*} e^{M L(n+N b) b^{\beta} / \Gamma(\beta+1)}}{1-M(c+\epsilon) e^{M L(n+N b) b^{\beta} / \Gamma(\beta+1)}}<\infty .
$$

Thus the proof of boundedness of the set $\Omega$ is complete.

SteP 2. $P$ maps bounded sets into equicontinuous sets in $C_{\delta}$.

For each constant $r>0$, let

$$
v \in C_{r}(\delta):=\left\{\phi \in C_{\delta}: \sup _{\delta \leq t \leq b}\|\phi(t)\| \leq r\right\} .
$$

Then $C_{r}(\delta)$ is a bounded closed convex set in $C_{\delta}$. Let $v \in C_{r}(\delta), \delta \leq t_{1}<$ $t_{2} \leq b$, and $\varepsilon>0$ be small. Note that

$$
\begin{aligned}
&\left\|F\left(s, \phi_{\widetilde{v}}\left(\sigma_{1}(s)\right), \ldots, \phi_{\widetilde{v}}\left(\sigma_{n}(s)\right), \int_{0}^{s} h\left(s, \tau, \phi_{\widetilde{v}}\left(\sigma_{n+1}(\tau)\right)\right) d \tau\right)\right\| \\
& \leq \| F\left(s, \phi_{\widetilde{v}}\left(\sigma_{1}(s)\right), \ldots, \phi_{\widetilde{v}}\left(\sigma_{n}(s)\right), \int_{0}^{s} h\left(s, \tau, \phi_{\widetilde{v}}\left(\sigma_{n+1}(\tau)\right)\right) d \tau\right) \\
&-F(s, 0, \ldots, 0)\|+\| F(s, 0, \ldots, 0) \| \\
& \leq L\left[\left\|\phi_{\widetilde{v}}\left(\sigma_{1}(s)\right)\right\|+\cdots+\left\|\phi_{\widetilde{v}}\left(\sigma_{n}(s)\right)\right\|\right. \\
&\left.+\left\|\int_{0}^{s} h\left(s, \tau, \phi_{\widetilde{v}}\left(\sigma_{n+1}(\tau)\right)\right) d \tau\right\|\right]+L_{1} \\
& \leq L\left[\left\|\phi_{\widetilde{v}}(s)\right\|+\cdots+\left\|\phi_{\widetilde{v}}(s)\right\|+\int_{0}^{s}\left[\left\|h\left(s, \tau, \phi_{\widetilde{v}}(\tau)\right)-h(s, \tau, 0)\right\|\right.\right. \\
&+\|h(s, \tau, 0)\|] d \tau]+L_{1} \\
& \leq L\left[n\left\|\phi_{\widetilde{v}}(s)\right\|+b\left[N \sup _{s \in[\delta, b]}\left\|\phi_{\widetilde{v}}(s)\right\|+N_{1}\right]\right]+L_{1} \\
& \leq L\left[(n+N b) \sup _{s \in[\delta, b]}\left\|\phi_{\widetilde{v}}(s)\right\|+b N_{1}\right]+L_{1} \\
& \leq L\left[(n+N b) r+b N_{1}\right]+L_{1}:=M^{* *} .
\end{aligned}
$$

We have

$$
\begin{aligned}
& \left\|P v\left(t_{2}\right)-P v\left(t_{1}\right)\right\| \\
& \leq\left\|\left[T\left(t_{2}\right)-T\left(t_{1}\right)\right]\left[x_{0}-g(\widetilde{v})\right]\right\| \\
& \quad+\frac{1}{\Gamma(\beta)} \int_{0}^{t_{1}-\varepsilon} \|\left[\left(t_{2}-s\right)^{\beta-1} T\left(t_{2}-s\right)-\left(t_{1}-s\right)^{\beta-1} T\left(t_{1}-s\right)\right]
\end{aligned}
$$




$$
\begin{aligned}
& \times F\left(s, \phi_{\widetilde{v}}\left(\sigma_{1}(s)\right), \ldots, \phi_{\widetilde{v}}\left(\sigma_{n}(s)\right), \int_{0}^{s} h\left(s, \tau, \phi_{\widetilde{v}}\left(\sigma_{n+1}(\tau)\right)\right) d \tau\right) \| d s \\
& +\frac{1}{\Gamma(\beta)} \int_{t_{1}-\varepsilon}^{t_{1}} \|\left[\left(t_{2}-s\right)^{\beta-1} T\left(t_{2}-s\right)-\left(t_{1}-s\right)^{\beta-1} T\left(t_{1}-s\right)\right] \\
& \times F\left(s, \phi_{\widetilde{v}}\left(\sigma_{1}(s)\right), \ldots, \phi_{\widetilde{v}}\left(\sigma_{n}(s)\right), \int_{0}^{s} h\left(s, \tau, \phi_{\widetilde{v}}\left(\sigma_{n+1}(\tau)\right)\right) d \tau\right) \| d s \\
& +\frac{1}{\Gamma(\beta)} \int_{t_{1}}^{t_{2}} \|\left(t_{2}-s\right)^{\beta-1} \\
& \times T\left(t_{2}-s\right) F\left(s, \phi_{\widetilde{v}}\left(\sigma_{1}(s)\right), \ldots, \phi_{\widetilde{v}}\left(\sigma_{n}(s)\right), \int_{0}^{s} h\left(s, \tau, \phi_{\widetilde{v}}\left(\sigma_{n+1}(\tau)\right)\right) d \tau\right) \| d s \\
& \leq\left\|\left[T\left(t_{2}\right)-T\left(t_{1}\right)\right]\left[x_{0}-g(\widetilde{v})\right]\right\| \\
& +\frac{M^{* *}}{\Gamma(\beta)}\left(\int_{0}^{t_{1}-\varepsilon}\left[\left(t_{1}-s\right)^{\beta-1}-\left(t_{2}-s\right)^{\beta-1}\right]\left\|T\left(t_{2}-s\right)\right\| d s\right. \\
& +\int_{0}^{t_{1}-\varepsilon}\left(t_{1}-s\right)^{\beta-1}\left\|T\left(t_{1}-s-\varepsilon\right)\right\|\left\|T\left(t_{2}-t_{1}+\varepsilon\right)-T(\varepsilon)\right\| d s \\
& +\int_{t_{1}-\varepsilon}^{t_{1}}\left[\left(t_{1}-s\right)^{\beta-1}-\left(t_{2}-s\right)^{\beta-1}\right]\left\|T\left(t_{2}-s\right)\right\| d s \\
& +\int_{t_{1}-\varepsilon}^{t_{1}}\left(t_{1}-s\right)^{\beta-1}\left\|T\left(t_{1}-s-\varepsilon\right)\right\|\left\|T\left(t_{2}-t_{1}+\varepsilon\right)-T(\varepsilon)\right\| d s \\
& \left.+\int_{t_{1}}^{t_{2}}\left(t_{2}-s\right)^{\beta-1}\left\|T\left(t_{2}-s\right)\right\| d s\right) \\
& \leq\left\|\left[T\left(t_{2}\right)-T\left(t_{1}\right)\right]\left[x_{0}-g(\widetilde{v})\right]\right\| \\
& +\frac{M^{* *}}{\Gamma(\beta)}\left(M \int_{0}^{t_{1}-\varepsilon}\left[\left(t_{1}-s\right)^{\beta-1}-\left(t_{2}-s\right)^{\beta-1}\right] d s\right. \\
& +M\left\|T\left(t_{2}-t_{1}+\varepsilon\right)-T(\varepsilon)\right\| \int_{0}^{t_{1}-\varepsilon}\left(t_{1}-s\right)^{\beta-1} d s \\
& +M \int_{t_{1}-\varepsilon}^{t_{1}}\left[\left(t_{1}-s\right)^{\beta-1}-\left(t_{2}-s\right)^{\beta-1}\right] d s \\
& \left.+M\left\|T\left(t_{2}-t_{1}+\varepsilon\right)-T(\varepsilon)\right\| \int_{t_{1}-\varepsilon}^{t_{1}}\left(t_{1}-s\right)^{\beta-1} d s+M \int_{t_{1}}^{t_{2}}\left(t_{2}-s\right)^{\beta-1} d s\right)
\end{aligned}
$$




$$
\begin{aligned}
\leq & \left\|\left[T\left(t_{2}\right)-T\left(t_{1}\right)\right]\left[x_{0}-g(\widetilde{v})\right]\right\|+\frac{M^{* *} M}{\beta \Gamma(\beta)}\left(\left[\left(t_{2}-t_{1}+\varepsilon\right)^{\beta}-\varepsilon^{\beta}+t_{1}^{\beta}-t_{2}^{\beta}\right]\right. \\
& +\left\|T\left(t_{2}-t_{1}+\varepsilon\right)-T(\varepsilon)\right\|\left(\varepsilon^{\beta}+t_{1}^{\beta}\right) \\
& +\left[\left(t_{2}-t_{1}\right)^{\beta}-\left(t_{2}-t_{1}-\varepsilon\right)^{\beta}+\varepsilon^{\beta}\right] \\
& \left.+\left\|T\left(t_{2}-t_{1}+\varepsilon\right)-T(\varepsilon)\right\| \varepsilon^{\beta}+\left(t_{2}-t_{1}\right)^{\beta}\right) .
\end{aligned}
$$

We see that $\left\|P v\left(t_{2}\right)-P v\left(t_{1}\right)\right\|$ tends to zero independently of $v \in C_{r}(\delta)$ as $t_{2}-t_{1} \rightarrow 0$, since the compactness of $T(t)$ for $t>0$ implies continuity in the uniform operator topology. Thus $P$ maps $C_{r}(\delta)$ into an equicontinuous family of functions.

STEP 3. The set $\left\{P(v)(t): v \in C_{r}(\delta)\right\}$ is relatively compact in $X$.

Let $\delta<t \leq s \leq b$ be fixed and $\varepsilon$ a real number satisfying $0<\varepsilon<t$. For $v \in C_{r}(\delta)$, we define

$$
\begin{aligned}
& \left(P_{\varepsilon} v\right)(t)=T(t)\left[x_{0}-g(\widetilde{v})\right]+\frac{1}{\Gamma(\beta)} \int_{0}^{t-\varepsilon}(t-s)^{\beta-1} \\
& \times T(t-s) F\left(s, \phi_{\widetilde{v}}\left(\sigma_{1}(s)\right), \ldots, \phi_{\widetilde{v}}\left(\sigma_{n}(s)\right), \int_{0}^{s} h\left(s, \tau, \phi_{\widetilde{v}}\left(\sigma_{n+1}(\tau)\right)\right) d \tau\right) d s \\
& =T(t)\left[x_{0}-g(\widetilde{v})\right]+\frac{T(\varepsilon)}{\Gamma(\beta)} \int_{0}^{t-\varepsilon}(t-s)^{\beta-1} \\
& \quad \times T(t-s-\varepsilon) F\left(s, \phi_{\widetilde{v}}\left(\sigma_{1}(s)\right), \ldots, \phi_{\widetilde{v}}\left(\sigma_{n}(s)\right), \int_{0}^{s} h\left(s, \tau, \phi_{\widetilde{v}}\left(\sigma_{n+1}(\tau)\right)\right) d \tau\right) d s .
\end{aligned}
$$

Using the compactness of $T(t)$ for $t>0$, we deduce that the set $\left\{\left(P_{\varepsilon} v\right)(t)\right.$ : $\left.v \in C_{r}(\delta)\right\}$ is precompact in $X$ for every $\varepsilon, 0<\varepsilon<t$. Moreover, for every $v \in C_{r}(\delta)$ we have

$$
\begin{aligned}
& \left\|(P v)(t)-\left(P_{\varepsilon} v\right)(t)\right\| \\
& \leq \frac{1}{\Gamma(\beta)} \int_{t-\varepsilon}^{t} \|(t-s)^{\beta-1} \\
& \quad \times T(t-s) F\left(s, \phi_{\widetilde{v}}\left(\sigma_{1}(s)\right), \ldots, \phi_{\widetilde{v}}\left(\sigma_{n}(s)\right), \int_{0}^{s} h\left(s, \tau, \phi_{\widetilde{v}}\left(\sigma_{n+1}(\tau)\right)\right) d \tau\right) \| d s \\
& \leq \frac{M M^{* *}}{\Gamma(\beta)} \int_{t-\varepsilon}^{t}(t-s)^{\beta-1} d s \leq \frac{M M^{* *}}{\beta \Gamma(\beta)} \varepsilon^{\beta} .
\end{aligned}
$$

Therefore, there are precompact sets arbitrarily close to the set $\{P v: v \in$ $\left.C_{r}(\delta)\right\}$. Hence the set $\left\{P v: v \in C_{r}(\delta)\right\}$ is precompact in $X$. It is easy to see that $P\left(C_{r}(\delta)\right)$ is uniformly bounded. Since we have shown $P\left(C_{r}(\delta)\right)$ is an 
equicontinuous collection, by the Arzelà-Ascoli theorem, it suffices to show that $P$ maps $C_{r}(\delta)$ into a precompact set in $X$.

SteP 4. $P: C_{\delta} \rightarrow C_{\delta}$ is continuous.

From (3.2) and (H1)-(H5), we deduce that for $v_{1}, v_{2} \in C_{r}(\delta), t \in(0, b]$, $\left\|\phi_{\widetilde{v}_{1}}(t)-\phi_{\widetilde{v}_{2}}(t)\right\|$ $\leq\left\|T(t)\left[g\left(\widetilde{v}_{1}\right)-g\left(\widetilde{v}_{2}\right)\right]\right\|+\frac{1}{\Gamma(\beta)} \int_{0}^{t} \|(t-s)^{\beta-1}$ $\times T(t-s)\left[F\left(s, \phi_{\widetilde{v}_{1}}\left(\sigma_{1}(s)\right), \ldots, \phi_{\widetilde{v}_{1}}\left(\sigma_{n}(s)\right), \int_{0}^{s} h\left(s, \tau, \phi_{\widetilde{v}_{1}}\left(\sigma_{n+1}(\tau)\right)\right) d \tau\right)\right.$ $\left.-F\left(s, \phi_{\widetilde{v}_{2}}\left(\sigma_{1}(s)\right), \ldots, \phi_{\widetilde{v}_{2}}\left(\sigma_{n}(s)\right), \int_{0}^{s} h\left(s, \tau, \phi_{\widetilde{v}_{2}}\left(\sigma_{n+1}(\tau)\right)\right) d \tau\right)\right] \| d s$ $\leq M\left\|g\left(\widetilde{v}_{1}\right)-g\left(\widetilde{v}_{2}\right)\right\|+\frac{L M}{\Gamma(\beta)} \int_{0}^{t}(t-s)^{\beta-1}$ $\times\left[\left\|\phi_{\widetilde{v}_{1}}\left(\sigma_{1}(s)\right)-\phi_{\widetilde{v}_{2}}\left(\sigma_{1}(s)\right)\right\|+\cdots+\left\|\phi_{\widetilde{v}_{1}}\left(\sigma_{n}(s)\right)-\phi_{\widetilde{v}_{2}}\left(\sigma_{n}(s)\right)\right\|\right.$ $\left.+\left\|\int_{0}^{s} h\left(s, \tau, \phi_{\widetilde{v}_{1}}\left(\sigma_{n+1}(\tau)\right)\right) d \tau-\int_{0}^{s} h\left(s, \tau, \phi_{\widetilde{v}_{2}}\left(\sigma_{n+1}(\tau)\right)\right) d \tau\right\|\right] d s$ $\leq M\left\|g\left(\widetilde{v}_{1}\right)-g\left(\widetilde{v}_{2}\right)\right\|+\frac{L M}{\Gamma(\beta)} \int_{0}^{t}(t-s)^{\beta-1}\left[\left\|\phi_{\widetilde{v}_{1}}(s)-\phi_{\widetilde{v}_{2}}(s)\right\|\right.$ $+\cdots+\left\|\phi_{\widetilde{v}_{1}}(s)-\phi_{\widetilde{v}_{2}}(s)\right\|$ $\left.+N \int_{0}^{s}\left\|\phi_{\widetilde{v}_{1}}\left(\sigma_{n+1}(\tau)\right)-\phi_{\widetilde{v}_{2}}\left(\sigma_{n+1}(\tau)\right)\right\| d \tau\right] d s$ $\leq M\left\|g\left(\widetilde{v}_{1}\right)-g\left(\widetilde{v}_{2}\right)\right\|+\frac{L M}{\Gamma(\beta)} \int_{0}^{t}(t-s)^{\beta-1}\left[n\left\|\phi_{\widetilde{v}_{1}}(s)-\phi_{\widetilde{v}_{2}}(s)\right\|\right.$ $\left.+N b\left\|\phi_{\widetilde{v}_{1}}(s)-\phi_{\widetilde{v}_{2}}(s)\right\|\right] d s$ $\leq M\left\|g\left(\widetilde{v}_{1}\right)-g\left(\widetilde{v}_{2}\right)\right\|$ $+\frac{L M(n+N b)}{\Gamma(\beta)} \int_{0}^{t}(t-s)^{\beta-1} \sup _{t \in J}\left\|\phi_{\widetilde{v}_{1}}(s)-\phi_{\widetilde{v}_{2}}(s)\right\| d s$.

Using again the Gronwall inequality, we see that, for $t, v_{1}, v_{2}$ as above,

$$
\sup _{t \in J}\left\|\phi_{\widetilde{v}_{1}}(t)-\phi_{\widetilde{v}_{2}}(t)\right\| \leq M e^{M L(n+N b) b^{\beta} / \Gamma(\beta+1)}\left\|g\left(\widetilde{v}_{1}\right)-g\left(\widetilde{v}_{2}\right)\right\|,
$$


which implies that

$$
\left\|P v_{1}-P v_{2}\right\| \leq M e^{M L(n+N b) b^{\beta} / \Gamma(\beta+1)}\left\|g\left(\widetilde{v}_{1}\right)-g\left(\widetilde{v}_{2}\right)\right\|,
$$

for all $t \in[\delta, b]$ and $v_{1}, v_{2} \in C_{r}(\delta)$. Therefore, $P$ is continuous.

These arguments enable us to conclude that $P$ is completely continuous. We can now apply Lemma 2.4 to conclude that $P$ has a fixed point $\widetilde{v}_{*} \in C_{\delta}$. Let $x=\phi_{\widetilde{v}_{*}}$. Then

$$
\begin{aligned}
& x(t)=T(t)\left[x_{0}-g\left(\widetilde{v}_{*}\right)\right]+\frac{1}{\Gamma(\beta)} \int_{0}^{t}(t-s)^{\beta-1} \\
& \quad \times T(t-s) F\left(s, x\left(\sigma_{1}(s)\right), \ldots, x\left(\sigma_{n}(s)\right), \int_{0}^{s} h\left(s, \tau, x\left(\sigma_{n+1}(\tau)\right)\right) d \tau\right) d s .
\end{aligned}
$$

Note that $x=\phi_{\widetilde{v}_{*}}=\left(P \widetilde{v}_{*}\right)(t)=\widetilde{v}_{*}, t \in[\delta, b]$. By (H5), we obtain

$$
g(x)=g\left(\widetilde{v}_{*}\right) \text {. }
$$

This implies, combined with (3.7), that $x(t)$ is a mild solution of the problem (1.1)-(1.2), and the proof of Theorem 3.1 is complete.

From the above proof of Theorem 3.1, we immediately obtain the following corollaries:

COROllary 3.2. Suppose that (H1)-(H5) are satisfied together with the following condition:

(H7) There exist constants $b_{1}$ and $b_{2}, \mu \in[0,1)$ such that

$$
\|g(\phi)\| \leq b_{1}+b_{2}\|\phi\|^{\mu}, \quad \phi \in C(J, X) .
$$

Then the nonlocal Cauchy problem (1.1)-(1.2) has at least one mild solution on $J$.

COROLlary 3.3. Suppose that (H1)-(H5) are satisfied together with the following condition:

(H8) There exist constants $c_{1}$ and $c_{2}$ such that

$$
\|g(\phi)\| \leq c_{1}+c_{2}\|\phi\|, \quad \phi \in C(J, X) .
$$

Then the nonlocal Cauchy problem (1.1)-(1.2) has at least one mild solution on $J$, provided that

$$
M c_{2} e^{M L(n+N b) b^{\beta} / \Gamma(\beta+1)}<1 .
$$

Remark 3.4. In $[\mathrm{AM}],[\mathrm{BP},[\mathrm{BL},[\mathrm{B},[\mathrm{BA},[\mathrm{D}], \mathrm{LL},[\mathrm{MN}], \mathrm{MG}$, $[\mathrm{N}],[\mathrm{NT}]$, the authors have discussed a related semilinear nonlocal Cauchy problem when $g$ satisfies Lipschitz-type conditions, or is convex and compact on a given ball. In this paper, we consider the case in which $g$ is continuous but without imposing severe compactness conditions or convexity. 
Remark 3.5. Conditions (H5)-(H6) on $g$ in the above theorem are an extension of the corresponding conditions in paper [LX].

4. Application. In this section, we give an example to illustrate our results. Consider the following fractional order partial integrodifferential equation:

$$
\begin{gathered}
D^{\beta} z(t, x)=\frac{\partial^{2}}{\partial x^{2}} z(t, x)+a_{1}(t) z(\sin t, x)+\sin z(t, x) \\
\quad+\frac{1}{1+t^{2}} \int_{0}^{t} a_{2}(s) z(\sin s, x) d s, \\
z(t, 0)=z(t, \pi)=0, \\
z(0, x)+\int_{\delta}^{1}[z(s, x)+\log (1+|z(s, x)|)] d s=z_{0}(x),
\end{gathered}
$$

where $0 \leq t \leq 1,0 \leq x \leq \pi, \delta>0, z_{0}(x) \in X=L^{2}([0, \pi])$ and $z_{0}(0)=$ $z_{0}(\pi)=0$.

Let $X=L^{2}([0, \pi])$ and let the operator $A: X \rightarrow X$ be given by $A u=u^{\prime \prime}$ with

$$
D(A):=\left\{u \in X: u^{\prime \prime} \in X, u(0)=u(\pi)=0\right\} .
$$

Then

$$
A u=\sum_{n=1}^{\infty} n^{2}\left(u, u_{n}\right) u_{n}, \quad u \in D(A),
$$

where $u_{n}(x)=\sqrt{2 / \pi} \sin (n x), n=1,2, \ldots$, is the orthogonal set of eigenfunctions of $A$. It is well known that $A$ is the infinitesimal generator of a compact analytic semigroup $T(t), t>0$, in $X$ and is given by

$$
T(t) u=\sum_{n=1}^{\infty} \exp \left(-n^{2} t\right)\left(u, u_{n}\right) u_{n}, \quad u \in X,
$$

where $T(t)$ satisfies hypothesis $(\mathrm{H} 1)$.

We assume that the functions $a_{i}(\cdot), i=1,2$, are continuous on $[0,1]$, and $l_{i}=\sup _{0 \leq s \leq 1}\left|a_{i}(s)\right|<1, i=1,2$.

Define $F:[0,1] \times X \times X \rightarrow X, h:[0,1] \times[0,1] \times X \rightarrow X$ and $g:$ $C([0,1], X) \rightarrow X$ by

$$
\begin{aligned}
F\left(t, z(\sigma(t)), \int_{0}^{t} h(t, s, z(\sigma(s))) d s\right)(x)= & a_{1}(t) z(\sin t, x)+\sin z(t, x) \\
& +\frac{1}{1+t^{2}} \int_{0}^{t} a_{2}(s) z(\sin s, x) d s
\end{aligned}
$$




$$
\int_{0}^{t} h(t, s, z(\sigma(s)))(x) d s=\frac{1}{1+t^{2}} \int_{0}^{t} a_{2}(s) z(\sin s, x)
$$

and

$$
g(z)(x)=\int_{\delta}^{1}[z(s, x)+\log (1+|z(s, x)|)], \quad z \in C([0,1], X) .
$$

Then equations (4.1-(4.3) take the abstract form (1.1)-(1.2). Moreover, we can choose some $\beta \in(0,1)$ such that

$$
M(1-\delta) e^{M\left(1+l_{1}+l_{2}\right)\left(1+l_{2}\right) / \Gamma(\beta+1)}<1 .
$$

Then condition (H6) in Section 2 holds, and all the other conditions stated in Theorem 3.1 are satisfied. Hence, problem (4.1)-(4.3) has a mild solution on $[0,1]$.

\section{References}

[AM] S. Aizicovici and M. McKibben, Existence results for a class of abstract nonlocal Cauchy problems, Nonlinear Anal. 39 (2000), 649-668.

[BP] K. Balachandran and J. Y. Park, Nonlocal Cauchy problem for abstract fractional semilinear evolution equations, Nonlinear Anal. 71 (2009), 4471-4475.

[BH] M. Benchohra, J. Henderson, S. K. Ntouyas and A. Ouahab, Existence results for fractional order functional differential equations with infinite delay, J. Math. Anal. Appl. 338 (2008), 1340-1350.

[B] L. Byszewski, Theorems about the existence and uniqueness of solutions of a semilinear evolution nonlocal Cauchy problem, ibid. 162 (1991), 494-505.

[BA] L. Byszewski and H. Akca, Existence of solutions of a semilinear functionaldifferential evolution nonlocal problem, Nonlinear Anal. 34 (1998), 65-72.

[BL] L. Byszewski and V. Lakshmikantham, Theorem about the existence and uniqueness of a solution of a nonlocal abstract Cauchy problem in a Banach space, Appl. Anal. 40 (1991), 11-19.

[D] K. Deng, Exponential decay of solutions of semilinear parabolic equations with nonlocal initial conditions, J. Math. Anal. Appl. 179 (1993), 630-637.

[DF] K. Diethelm and N. J. Ford, Analysis of fractional differential equations, ibid. 265 (2002), 229-248.

[DG] J. Dugundji and A. Granas, Fixed Point Theory, Monografie Mat. 61, PWN, Warszawa, 1982.

[E] M. M. El-Borai, Semigroups and some nonlinear fractional differential equations, Appl. Math. Comput. 149 (2004), 823-831.

[ES] A. M. A. El-Sayed, Nonlinear functional differential equations of arbitrary order, Nonlinear Anal. 33 (1998), 181-186.

[EI] A. M. A. El-Sayed and A.-G. Ibrahim, Set-valued integral equations of fractionalorders, Appl. Math. Comput. 118 (2001), 113-121.

[H] R. Hilfer, Applications of Fractional Calculus in Physics, World Sci., Singapore, 2000 .

[IM] R. W. Ibrahim and S. Momani, On the existence and uniqueness of solutions of a class of fractional differential equations, J. Math. Anal. Appl. 334 (2007), 1-10. 
[JA] O. K. Jaradat, A. Al-Omari and S. Momani, Existence of the mild solution for fractional semilinear initial value problem, Nonlinear Anal. 69 (2008), 3153-3159.

[L] V. Lakshmikantham, Theory of fractional functional differential equations, ibid. 60 (2008), 3337-3343.

[LV] V. Lakshmikantham and A. S. Vatsala, Basic theory of fractional differential equations, ibid. 69 (2008), 2677-2682.

[LX] J. Liang, J. H. Liu and T. J. Xiao, Nonlocal Cauchy problems governed by compact operator families, ibid. 57 (2004), 183-189.

[LL] Y. Lin and J. H. Liu, Semilinear integrodifferential equations with nonlocal Cauchy problem, ibid. 26 (1996), 1023-1033.

[MR] K. S. Miller and B. Ross, An Introduction to the Fractional Calculus and Differential Equations, Wiley, New York, 1993.

[MN] G. M. Mophou and G. M. N'Guérékata, On integral solutions of some nonlocal fractional differential equations with nondense domain, Nonlinear Anal. 70 (2009), $4668-4675$.

[MG] -, - Existence of the mild solution for some fractional differential equations with nonlocal conditions, Semigroup Forum 79 (2009), 315-322.

[M] M. Muslim, Existence and approximation of solutions to fractional differential equations, Math. Comput. Model. 49 (2009), 1164-1172.

[N] G. M. N'Guérékata, A Cauchy problem for some fractional abstract differential equation with nonlocal conditions, Nonlinear Anal. 70 (2009), 1873-1876.

[NT] S. K. Ntouyas and P. Ch. Tsamatos, Global existence for semilinear evolution equations with nonlocal conditions, J. Math. Anal. Appl. 210 (1997), 679-687.

[P] I. Podlubny, Fractional Differential Equations, Math. Sci. Engrg. 198, Academic Press, San Diego, 1999.

[SK] S. G. Samko, A. A. Kilbas and O. I. Marichev, Fractional Integrals and Derivatives. Theory and Applications, Gordon and Breach, 1993.

[Y] K. Yosida, Functional Analysis, 6th ed., Springer, Berlin, 1980.

[YG] C. Yu and G. Gao, Existence of fractional differential equations, J. Math. Anal. Appl. 310 (2005), 26-29.

Zuomao Yan

Department of Mathematics

Hexi University

Zhangye, Gansu 734000, P.R. China

E-mail: yanzuomao@163.com

Received 3.7.2009

and in final form 31.8.2009 
